\title{
Predictors of admission and shunt revision during emergency department visits for shunt-treated adult patients with idiopathic intracranial hypertension
}

\author{
*Eric W. Sankey, MD, Benjamin D. Elder, MD, PhD, ${ }^{1}$ Ann Liu, MD, ${ }^{1}$ Kathryn A. Carson, ScM, ${ }^{2}$ \\ C. Rory Goodwin, MD, PhD, Ignacio Jusué-Torres, MD, ${ }^{1}$ and Daniele Rigamonti, MD ${ }^{1}$ \\ 'Department of Neurosurgery, Johns Hopkins University School of Medicine; and 2Department of Epidemiology, Johns Hopkins \\ Bloomberg School of Public Health, Baltimore, Maryland
}

\begin{abstract}
OBJECTIVE Factors associated with emergency department admission and/or shunt revision for idiopathic intracranial hypertension $(\mathrm{IIH})$ are unclear. In this study, the associations of several factors with emergency department admission and shunt revision for $\mathrm{IIH}$ were explored.

METHODS The authors performed a retrospective review of 31 patients (169 total emergency department visits) who presented to the emergency department for IIH-related symptoms between 2003 and 2015. Demographics, comorbidities, symptoms, IIH diagnosis and treatment history, ophthalmological examination, diagnostic lumbar puncture (LP), imaging findings, and data regarding admission and management decisions were collected. Multivariable general linear models regression analysis was performed to assess the predictive factors associated with admission and shunt revision.
\end{abstract}

RESULTS Thirty-one adult patients with a history of shunt placement for IIH visited the emergency department a total of 169 times for IIH-related symptoms, with a median of 3 visits (interquartile range 2-7 visits) per patient. Five patients had more than 10 emergency department visits. Baseline factors associated with admission included male sex (OR 10.47, $95 \% \mathrm{Cl} 2.13-51.56 ; p=0.004)$ and performance of an LP (OR 3.10, 95\% Cl 1.31-7.31; $p=0.01)$. Contrastingly, older age at presentation (OR $0.94,95 \% \mathrm{Cl} 0.90-0.99 ; p=0.01$ ), and a greater number of prior emergency department visits (OR $0.94,95 \% \mathrm{Cl} 0.89-0.99 ; \mathrm{p}=0.02$ ) were slightly protective against admission. The presence of papilledema (OR 11.62, $95 \% \mathrm{Cl} 3.20-42.16$; $p<0.001)$, Caucasian race (OR 40.53, 95\% Cl 2.49-660.09 $p=0.009$ ), and systemic hypertension (OR 7.73, 95\% Cl 1.11-53.62; $p=0.03$ ) were independent risk factors for shunt revision. In addition, a greater number of prior emergency department visits $(\mathrm{OR} 0.86,95 \% \mathrm{Cl} 0.77-0.96 ; p=0.009)$ and older age at presentation $(\mathrm{OR} 0.93,95 \%$ $\mathrm{Cl} 0.87-0.99 ; p=0.02$ ) were slightly protective against shunt revision, while there was suggestive evidence that presence of a programmable shunt $(\mathrm{OR} 0.23,95 \% \mathrm{Cl} 0.05-1.14 ; \mathrm{p}=0.07)$ was a protective factor against shunt revision. Of note, location of the proximal catheter in the ventricle or lumbar subarachnoid space was not significantly associated with admission or shunt revision in the multivariable analyses.

CONCLUSIONS The decision to admit a shunt-treated patient from the emergency department for symptoms related to $\mathrm{IIH}$ is challenging. Knowledge of factors associated with the need for admission and/or shunt revision is required. In this study, factors such as male sex, younger age at presentation, lower number of prior emergency department visits, and performance of a diagnostic LP were independent predictors of admission. In addition, papilledema was strongly predictive of the need for shunt revision, highlighting the importance of an ophthalmological examination for shunt-treated adults with $\mathrm{IIH}$ who present to the emergency department.

https://thejns.org/doi/abs/10.3171/2016.5.JNS151303

KEY WORDS shunt; pseudotumor cerebri; idiopathic intracranial hypertension; hospital admission; emergency department; hydrocephalus

ABBREVIATIONS BMI = body mass index; ICP = intracranial pressure; $I I \mathrm{H}=$ idiopathic intracranial hypertension; $I Q R=$ interquartile range; $L P=$ lumbar puncture. SUBMITTED June 5, 2015. ACCEPTED May 18, 2016.

INCLUDE WHEN CITING Published online September 23, 2016; DOI: 10.3171/2016.5.JNS151303.

* Drs. Sankey and Elder contributed equally to this work. 
I DIOPATHIC intracranial hypertension (IIH), also known as pseudotumor cerebri, is a diagnosis of exclusion for patients with the clinical syndrome of increased intracranial pressure (ICP), without ventriculomegaly, in the absence of an identifiable causative factor such as a spaceoccupying or vascular lesion. ${ }^{2}$ IIH was first described in 1937 by Dandy, ${ }^{6}$ and the diagnostic criteria were revised by Friedman and Jacobson in 2002 to the following 6 conditions: 1) presenting symptoms may only represent generalized intracranial hypertension or papilledema; 2) presenting signs may only represent generalized intracranial hypertension or papilledema; 3 ) documentation of elevated ICP measured in the lateral decubitus position; 4) normal CSF composition; 5) no evidence of hydrocephalus, mass, structural, or vascular lesion on MRI or contrast-enhanced CT for typical patients, and MRI and MR venography for all others; and 6) no other cause of intracranial hypertension identified. ${ }^{9}$ Of note, Friedman and Jacobson considered an obese female of childbearing age as a typical patient with $\mathrm{IIH}$, while atypical features of IIH included male sex, a low to normal body mass index (BMI), and extremes of age. ${ }^{9}$

The reported incidence of IIH in the general population ranges from 0.5 to $2.0 / 100,000$, with an increase to 19/100,000 among overweight patients. ${ }^{1,11}$ As the prevalence of obesity increases in the western world, the number of individuals with IIH continues to increase..$^{10,11,16} \mathrm{Up}$ to $65 \%$ of patients with a diagnosis of $\mathrm{IIH}$ repeatedly visit the emergency department throughout the course of their disease $^{12-15}$ and place a substantial financial burden on the United States health care system. ${ }^{10}$ Yet, relatively limited attention has been given toward its impact. ${ }^{10}$

The most common presenting symptoms of IIH include headache, occurring in $68 \%-98 \%$ of patients, followed by visual disturbance, affecting $57 \%-72 \%$ of patients. ${ }^{2}$ Obese women of reproductive age are most commonly affected, with a reported female to male ratio of $4: 1$ to $15: 1 .^{2}$ However, men may be at a greater risk of severe vision loss., ${ }^{1,4}$ Despite the fact that no consensus exists on how patients should be monitored and treated, ${ }^{3}$ the majority of patients are managed conservatively with observation and medication, such as acetazolamide. ${ }^{3}$ Surgery, such as shunt placement, is reserved for patients whose condition is refractory to medical treatment or if vision loss is severe at diagnosis. ${ }^{8,12}$

While few studies have focused on the presentation of $\mathrm{IIH}$ in the emergency department, ${ }^{12}$ Koerner and Friedman recently reported that $40 \%$ of emergency department visits related to IIH resulted in admission, the majority of which were due to either recurrent symptoms or postoperative complications. ${ }^{13}$ Determining which patients should be admitted from the emergency department versus discharged and managed as outpatients is challenging, particularly for shunt-treated patients with potential crossover between symptoms related to IIH and shunt malfunction. Yet, the factors that result in admission from the emergency department have not been identified. Knowledge of these factors, particularly in shunt-treated patients, is needed to aid clinicians and surgeons in the appropriate management of patients presenting to the emergency department for symptoms related to IIH.
In this study, the associations of several factors with emergency department admission and/or shunt revision for IIH-related symptoms in adults with a history of shunt placement for IIH were explored.

\section{Methods \\ Patient Population}

The clinical data of 31 adult patients with a history of shunt placement for IIH who presented to the emergency department a total of 169 times for IIH-related symptoms between 2003 and 2015 were retrospectively reviewed. The study protocol was approved by the institutional review board. All patient data were obtained from the senior author's prospectively maintained database of 137 adult patients with shunt-treated IIH. emergency department visits prior to initial shunt placement (17 visits), emergency department visits in which patients left before being fully evaluated (2 visits), and emergency department visits unrelated to IIH (66 visits) were excluded from analysis. Demographic data regarding sex, race, age at diagnosis of IIH and emergency department visit, BMI, comorbidities, smoking history, acetazolamide use, and shunting history were collected. Data regarding type and duration of symptoms, ophthalmological examination, diagnostic lumbar puncture (LP) and imaging findings (i.e., CT, shunt survey, and shunt patency examination) were also assessed. In addition, information regarding admission and management changes was evaluated. Management changes were grouped by therapeutic CSF drainage via LP, medication change, shunt reprograming, and shunt revision. Information regarding length of stay and additional complications for admitted visits was also collected.

\section{Statistical Analysis}

Baseline demographic and clinical characteristics were summarized using frequency and percentage or median and interquartile range (IQR). Management changes in the emergency department were compared across admission groups using Fisher's exact test or the Wilcoxon rank-sum test. To account for repeated emergency department visits for some patients, general linear models regression analysis with a logit link was used. An exchangeable correlation structure was assumed. Univariable analyses of the possible predictors for the outcomes of admission and shunt revision were performed. Multivariable regression analysis including all possible predictors was then performed. To develop more parsimonious models, nonsignificant covariates were dropped from the model one at a time until no covariates with a $\mathrm{p}<0.10$ remained. Analysis was performed using SAS (version 9.3, SAS Institute, Inc.). Odds ratios are presented with $95 \%$ confidence intervals. Statistical significance was determined by a two-sided $\mathrm{p}$ value $<0.05$.

\section{Results}

\section{Baseline Characteristics}

Over a 12-year period, 31 patients with a diagnosis of IIH visited the emergency department at our institution a total of 169 times for IIH-related symptoms. Of these 
visits, 38 (22\%) resulted in admission and 131 (78\%) resulted in discharge from the emergency department, with a median of 3 visits (IQR 2-7 visits) and 1 admission (IQR 0-2 admissions) per patient. Five patients had more than $10 \mathrm{IIH}$-related emergency department visits. The majority of patients were female (27 patients, $87 \%$ ) and Caucasian (21 patients, $68 \%$ ). The median age at diagnosis of IIH was 28 years (IQR 22-35 years), with a median age of 31 years (IQR 25-46 years) at first presentation to the emergency department for IIH-related symptoms. The median BMI was $35 \mathrm{~kg} / \mathrm{m}^{2}$ (IQR $\left.27-43 \mathrm{~kg} / \mathrm{m}^{2}\right)$. Migraine and systemic hypertension were present in $10(32 \%)$ patients each, and represented the most common comorbidities. Six (19\%) patients had a history of smoking. Approximately onethird of the patients (9 patients, 29\%) were receiving acetazolamide at the time of at least one of their emergency department visits (Table 1).

All patients were treated with CSF shunting prior to their initial presentation to the emergency department. The proximal catheter was placed in the lateral ventricle in $19(61 \%)$ patients and lumbar subarachnoid space in $10(32 \%)$ patients. Two $(6 \%)$ patients had their shunts removed shortly before their initial emergency department visit. Shunts were programmable in 19 (61\%) patients. In addition, an antisiphon device was present in 12 (39\%) patients and a horizontal-vertical (HV) valve in $4(13 \%)$ patients (Table 1).

\section{Chief Complaints and Presenting Symptoms}

The most common chief complaint when presenting to the emergency department was severe headache (133 visits, 79\%). After headache, the second most common symptom was nausea/vomiting, occurring in $93(55 \%)$ visits, followed in decreasing frequency by visual deficits (62 visits, $37 \%$ ), dizziness (17 visits, $10 \%$ ), fever/chills (10 visits, $6 \%$ ), tinnitus (4 visits, $2 \%$ ), and urinary dysfunction (2 visits, 1\%). Of note, visual deficits included diminished visual acuity/blurred vision in $58(34 \%)$ visits and visual field cuts in $5(3 \%)$ visits. A shunt malfunction/infection was discovered in a total of $9(5 \%)$ visits. Patients had symptoms for a median of 3 days (IQR $1-5$ days) prior to presenting to the emergency department.

\section{Diagnostic Studies and Findings}

An ophthalmology examination was performed in the majority of visits resulting in admission (20 visits, $53 \%)$ as well as discharge (74 visits, $56 \%$; $\mathrm{p}=0.71$ ), with papilledema observed in $14(8 \%)$ visits. A head CT was obtained in $131(78 \%)$ visits, with new pathology observed via CT on 6 (5\%) scans. A shunt survey was obtained in 105 (62\%) visits, with new pathology observed in $4(4 \%)$ shunt surveys. A shunt patency examination was performed in $10(6 \%)$ visits, with new pathology observed in 4 (40\%) shunt patency examinations. Lastly, a diagnostic LP was obtained in $19(50 \%)$ visits, resulting in admission compared with $41(31 \%)$ visits leading to discharge $(p=0.05)$, with a median opening pressure of $24 \mathrm{~cm} \mathrm{H}_{2} \mathrm{O}$ (IQR 18-27 $\mathrm{cm} \mathrm{H}_{2} \mathrm{O}$ ) in the admitted group and $30 \mathrm{~cm} \mathrm{H}_{2} \mathrm{O}$ (IQR 22-33 cm $\left.\mathrm{H}_{2} \mathrm{O}\right)$ in the discharged group $(\mathrm{p}=0.048)$ (Table 2).
TABLE 1. Patient demographics

\begin{tabular}{|c|c|}
\hline Patient Characteristics & All Patients $(n=31)$ \\
\hline \multicolumn{2}{|l|}{ Median age at diagnosis, yrs } \\
\hline Median & 28 \\
\hline IQR & $22-35$ \\
\hline \multicolumn{2}{|c|}{ Median age at initial presentation, yrs* } \\
\hline Median & 31 \\
\hline IQR & $25-46$ \\
\hline Female sex & $27(87)$ \\
\hline \multicolumn{2}{|l|}{ Race } \\
\hline Caucasian & $21(68)$ \\
\hline African American & $10(32)$ \\
\hline \multicolumn{2}{|l|}{$\mathrm{BMI}, \mathrm{kg} / \mathrm{m}^{2}$} \\
\hline Median & 35 \\
\hline IQR & $27-43$ \\
\hline \multicolumn{2}{|l|}{ Comorbidities } \\
\hline Migraines & $10(32)$ \\
\hline Systemic hypertension & $10(32)$ \\
\hline Diabetes/hyperglycemia & $3(10)$ \\
\hline Coagulopathy & $2(6)$ \\
\hline Cardiovascular disease & $2(6)$ \\
\hline Hyperlipidemia & $3(10)$ \\
\hline Gastrointestinal dysfunction & $1(3)$ \\
\hline Renal dysfunction & $0(0)$ \\
\hline COPD & $1(3)$ \\
\hline Smoking history & $6(19)$ \\
\hline Acetazolamide use & $9(29)$ \\
\hline \multicolumn{2}{|l|}{ Proximal catheter location } \\
\hline Ventricular & $19(61)$ \\
\hline Lumbar & $10(32)$ \\
\hline None & $2(6)$ \\
\hline Programmable shunt & $19(61)$ \\
\hline Antisiphon device & $12(39)$ \\
\hline Horizontal-vertical valve & $4(13)$ \\
\hline
\end{tabular}

Values are presented as the number of patients (\%) unless indicated otherwise. COPD = chronic obstructive pulmonary disease .

* Age at initial presentation calculated based on only emergency department visits related to $\mathrm{IH}$.

\section{Indications, Length of Stay, and Complications of Admission}

The most common indication for admission from the emergency department was severe, uncontrollable headache (19 visits, 50\%), followed by shunt malfunction/infection (10 visits, 26\%). Other indications for admission included postoperative complication (e.g., wound dehiscence $[3,8 \%]$ and catheter-induced urinary tract infection $[1,3 \%])$, acute changes in vision $(2,5 \%)$, abdominal/back pain near the shunt catheter $(2,5 \%)$, and need for LP performed under fluoroscopy $(1,3 \%)$. The median length of stay in the admitted group was 3 days (IQR 2-4 days). One (3\%) complication was encountered during admission related to shunt infection after shunt revision with insertion of a lumboatrial shunt. 
TABLE 2. Lumbar puncture and management changes performed during emergency department visit and/or admission

\begin{tabular}{lccc}
\hline \multicolumn{1}{c}{ Management Change } & $\begin{array}{c}\text { Admitted ED } \\
\text { Visits }(\mathrm{n}=38)\end{array}$ & $\begin{array}{c}\text { Discharged ED } \\
\text { Visits }(\mathrm{n}=131)\end{array}$ & $\mathrm{p}$ Value \\
\hline Diagnostic lumbar puncture & $19(50)$ & $41(31)$ & 0.05 \\
\hline Opening pressure, $\mathrm{cm} \mathrm{H}_{2} \mathrm{O}$ & & & $\mathbf{0 . 0 4 8}$ \\
\hline Median & 24 & 30 & \\
\hline $\mathrm{IQR}$ & $18-27$ & $22-33$ & \\
\hline Any change in treatment & $16(42)$ & $10(8)$ & $<0.001$ \\
\hline Shunt revision* & $12(75)$ & $2(20)$ & $\mathbf{0 . 0 1}$ \\
\hline Shunt reprogramming* $^{*}$ & $0(0)$ & $3(30)$ & $\mathbf{0 . 0 4 6}$ \\
\hline Medication change $^{*}$ & $4(25) \dagger$ & $4(40)$ & 0.66 \\
\hline Therapeutic CSF drainage $^{*}$ & $1(6) \dagger$ & $1(10)$ & 0.99 \\
\hline
\end{tabular}

Values are presented as the number of patients (\%) unless indicated otherwise. Boldface type indicates statistical significance.

$\mathrm{ED}=$ emergency department.

* Percentages for each management change based upon the total number of management changes per group.

$\dagger$ A combination of therapeutic CSF drainage via lumbar puncture and new valproate prescription was provided during 1 visit.

\section{Predictors of Admission}

On univariable general linear models logistic regression analysis, several variables were found to be positive predictors of admission, including male sex (OR 10.04, 95\% CI 1.27-79.37; $\mathrm{p}=0.03$ ), papilledema (OR 3.82, 95\% CI 1.06-13.79; $\mathrm{p}=0.04)$, and performance of a diagnostic LP (OR 1.86, 1.19-2.89; $\mathrm{p}=0.006)$. Conversely, factors associated with a lower likelihood of admission on univariable analysis included presence of a programmable shunt (OR 0.30, 95\% CI 0.09-0.99; $\mathrm{p}=0.048)$ and greater number of prior emergency department visits (OR 0.93, 95\% CI 0.89-0.97; p < 0.001) (Table 3).

On multivariable analysis, male sex (OR 10.47, 95\% CI 2.13-51.56; $p=0.004$ ) and performance of an LP (OR 3.10, 95\% CI 1.31-7.31; $p=0.01$ ) were independent predictors of admission, while older age at presentation (OR 0.94, 95\% CI $0.90-0.99 ; p=0.01$ ) and a greater number of prior emergency department visits (OR 0.94, 95\% CI 0.89-0.99; $\mathrm{p}=$ 0.02 ) were slightly protective against admission (Table 4).

\section{Management Changes}

Treatment changes were made during $16(42 \%)$ visits in the admitted group and $10(8 \%)$ visits in the discharged group ( $\mathrm{p}<0.001)$. In the admitted group, treatment changes entailed $12(75 \%)$ emergent shunt revisions, 1 $(6 \%)$ new acetazolamide prescription, 1 (6\%) short course of steroids, $1(6 \%)$ new amitriptyline prescription, and a combination of therapeutic CSF drainage via LP (6\%) and new valproate prescription (6\%) during 1 visit. In the discharged group, changes in treatment included 4 (40\%) changes in acetazolamide dosage, $3(30 \%)$ shunt setting reprogrammings, $1(10 \%)$ therapeutic CSF drainage via LP, and scheduling for 2 (20\%) later elective shunt revisions. The admitted group was significantly more likely to undergo shunt revision $(\mathrm{p}=0.01)$, while a greater frequency of shunt reprogramming was performed in the discharged group $(\mathrm{p}=0.046)$ (Table 2$)$.
TABLE 3. Univariable general linear models logistic regression analysis for admission

\begin{tabular}{lccc}
\hline \multicolumn{1}{c}{ Variable } & OR & $95 \% \mathrm{Cl}$ & $\mathrm{p} \mathrm{Value}$ \\
\hline Age at presentation, yrs & 0.97 & $0.92-1.02$ & 0.23 \\
\hline Male sex & 10.04 & $1.27-79.37$ & $\mathbf{0 . 0 3}$ \\
\hline Caucasian race & 3.19 & $0.96-10.67$ & 0.06 \\
\hline Systemic hypertension & 0.34 & $0.11-1.06$ & 0.06 \\
\hline Programmable shunt & 0.30 & $0.09-0.99$ & $\mathbf{0 . 0 4 8}$ \\
\hline No. of prior ED visits & 0.93 & $0.89-0.97$ & $<0.001$ \\
\hline Papilledema & 3.82 & $1.06-13.79$ & $\mathbf{0 . 0 4}$ \\
\hline $\begin{array}{l}\text { Lumbar puncture } \\
\text { Proximal ventricular catheter }\end{array}$ & 1.86 & $1.19-2.89$ & $\mathbf{0 . 0 0 6}$ \\
\hline$\quad$ vs none & 0.68 & $0.16-2.99$ & 0.61 \\
\hline Distal lumbar catheter vs none & 1.69 & $0.51-5.55$ & 0.39 \\
\hline
\end{tabular}

Boldface type indicates statistical significance.

\section{Predictors of Shunt Revision}

A subanalysis of the factors associated with shunt revision was also performed. Emergency department visits that occurred while the patient did not have a shunt $(\mathrm{n}=$ 15 ) were excluded for this outcome. No risk factors were found to be predictive of shunt revision on univariable analysis. Conversely, factors that were potentially protective against shunt revision included the presence of a programmable shunt (OR 0.17, 95\% CI 0.04-0.63; $\mathrm{p}=0.009$ ), a greater number of prior emergency department visits (OR 0.97, 95\% CI 0.95-0.99; $\mathrm{p}=0.002$ ), and use of a ventricular versus lumbar shunt (OR 0.35, 95\% CI 0.12-1.00; $\mathrm{p}=0.049)$ (Table 5).

On multivariable analysis of the total number of emergency department visits in shunt-treated patients (n $=154)$, the presence of papilledema (OR 11.62, 95\% CI $3.20-42.16 ; \mathrm{p}<0.001)$, Caucasian race (OR 40.53, 95\% CI 2.49-660.09; $\mathrm{p}=0.009$ ), and systemic hypertension (OR $7.73,95 \%$ CI 1.11-53.62; $p=0.04$ ) were independent risk factors for shunt revision. Contrastingly, a greater number of prior emergency department visits (OR 0.86, 95\% CI $0.77-0.96 ; \mathrm{p}=0.009)$ and older age at presentation (OR $0.93,95 \%$ CI $0.87-0.99 ; \mathrm{p}=0.02$ ) were slightly protective against shunt revision, while the presence of a programmable shunt (OR 0.23, 95\% CI 0.05-1.14; $\mathrm{p}=0.07$ ), though not significant, provided suggestive evidence of being a protective factor against shunt revision (Table 6). Of note, location of the proximal catheter in the ventricle or lumbar subarachnoid space was not significantly associated with admission or shunt revision in the multivariable analyses.

\section{Discussion}

Idiopathic intracranial hypertension is a complex disorder of unknown etiology that results in elevated ICP without ventriculomegaly. ${ }^{2}$ Patients often present to the emergency department for recurrent symptoms, postoperative complications, or shunt malfunction/infection, ${ }^{12,13}$ with the most common chief complaint of severe headache (79\%), followed by shunt malfunction/infection (5\%) in our study. Emergency department visits for IIH are likely to increase with an increasing number of patients diag- 
TABLE 4. Multivariable general linear models logistic regression analysis for admission

\begin{tabular}{lrll}
\hline \multicolumn{1}{c}{ Variable } & \multicolumn{1}{c}{ OR } & \multicolumn{1}{c}{$95 \% \mathrm{Cl}$} & $\mathrm{p}$ Value \\
\hline Age at presentation, yrs & 0.94 & $0.90-0.99$ & $\mathbf{0 . 0 1}$ \\
\hline Male sex & 10.47 & $2.13-51.56$ & $\mathbf{0 . 0 0 4}$ \\
\hline No. of prior ED visits & 0.94 & $0.89-0.99$ & $\mathbf{0 . 0 2}$ \\
\hline Papilledema & 2.89 & $0.82-10.14$ & 0.098 \\
\hline Lumbar puncture & 3.10 & $1.31-7.31$ & $\mathbf{0 . 0 1}$ \\
\hline
\end{tabular}

Boldface type indicates statistical significance.

nosed with IIH each year, concomitant with the rise in obesity. ${ }^{10,11,16}$ Despite this fact, limited attention has been paid to this unique and challenging patient population. In this study, the associations of several factors with emergency department admission and shunt revision for IIHrelated symptoms in shunt-treated adults were explored. Several factors were found to be associated with a higher likelihood of admission, including younger age at presentation, male sex, fewer prior emergency department visits, and performance of an LP. Interestingly, younger age at presentation and fewer prior emergency department visits were also predictive of shunt revision. Other independent risk factors for shunt revision in our study included the presence of papilledema on ophthalmological examination, Caucasian race, and systemic hypertension. To our knowledge, this is the first study to evaluate the factors associated with admission from the emergency department and shunt revision for shunt-treated adults presenting with symptoms related to IIH.

Patients frequently and repeatedly present to the emergency department for symptoms related to $\mathrm{IIH}$, with a median of 3 visits (range 1-46 visits) per patient in our study. According to Koerner and Friedman's study of 137 encounters from 51 patients over an 11-year period, the number of emergency department visits more than doubled over their study period, with a mean of 2.7 visits per patient. ${ }^{13}$ Forty percent of encounters in their study resulted in hospital admission, compared with $22 \%$ in our study, and like the emergency department visits in our study, were most commonly the result of recurrent symptoms

TABLE 5. Univariable general linear models logistic regression analysis for shunt revision

\begin{tabular}{lccc}
\hline \multicolumn{1}{c}{ Variable } & OR & $95 \% \mathrm{Cl}$ & $\mathrm{p} \mathrm{Value}$ \\
\hline Age at presentation, yrs & 0.94 & $0.88-1.01$ & 0.08 \\
\hline Male sex & 3.46 & $0.55-21.86$ & 0.19 \\
\hline Caucasian race & 8.62 & $0.91-81.54$ & 0.06 \\
\hline Systemic hypertension & 0.37 & $0.06-2.17$ & 0.27 \\
\hline Programmable shunt & 0.17 & $0.04-0.63$ & $\mathbf{0 . 0 0 9}$ \\
\hline No. of prior ED visits & 0.97 & $0.95-0.99$ & $\mathbf{0 . 0 0 2}$ \\
\hline Papilledema & 2.95 & $0.93-9.40$ & 0.07 \\
\hline Lumbar puncture & 0.80 & $0.35-1.80$ & 0.58 \\
\hline Proximal vs distal catheter & 0.35 & $0.12-1.00$ & $\mathbf{0 . 0 4 9}$ \\
\hline
\end{tabular}

Emergency department visits that occurred while a patient did not have a shunt $(n=15)$ were excluded for this outcome. Boldface type indicates statistical significance.
TABLE 6. Multivariable general linear models logistic regression analysis for shunt revision

\begin{tabular}{lrcc}
\hline \multicolumn{1}{c}{ Variable } & \multicolumn{1}{c}{ OR } & \multicolumn{1}{c}{$95 \% \mathrm{Cl}$} & $\mathrm{p}$ Value \\
\hline Age at presentation, yrs & 0.93 & $0.87-0.99$ & $\mathbf{0 . 0 2}$ \\
\hline Caucasian race & 40.53 & $2.49-660.09$ & $\mathbf{0 . 0 0 9}$ \\
\hline Systemic hypertension & 7.73 & $1.11-53.62$ & $\mathbf{0 . 0 4}$ \\
\hline Programmable shunt & 0.23 & $0.05-1.14$ & 0.07 \\
\hline No. of prior ED visits & 0.86 & $0.77-0.96$ & $\mathbf{0 . 0 0 9}$ \\
\hline Papilledema & 11.62 & $3.20-42.16$ & $<\mathbf{0 . 0 0 1}$ \\
\hline
\end{tabular}

Emergency department visits that occurred while a patient did not have a shunt $(n=15)$ were excluded for this outcome. Boldface type indicates statistical significance.

from IIH (43\%) or postoperative complications $(26 \%) .{ }^{13}$ As in our study, shunt revision was the most commonly performed operation in admitted patients..$^{13}$ Furthermore, in Friesner and colleagues' study of the prevalence and the direct and indirect costs of IIH using a prevalencebased model, the authors found that IIH patients had a hospital admission rate of $38 \% .^{10}$ In addition, the total hospital costs per IIH admission were 4 times greater than the population-based controls, with total economic costs of IIH patients exceeding \$444 million in $2007 .{ }^{10}$ Likewise, Jones et al. studied the presentation of IIH in the emergency department and found that $65 \%$ of patients in their study repeatedly returned to the emergency department for long-term complications related to IIH, with $12 \%$ of visits resulting in hospital admission. ${ }^{12}$ However, the authors of these studies did not evaluate the factors that were associated with the decision to admit patients from the emergency department.

Our results suggest that, though uncommon with IIH, male sex may be one of the most important risk factors associated with admission from the emergency department in patients with IIH. In our study, males were 10.47 times more likely to be admitted than female patients. Several studies have found that males with IIH may have a worse prognosis and have up to a 2 times greater risk of severe vision loss compared with female patients. ${ }^{4,7}$ Bruce et al. suggested that this risk might be due to the assumption that men with IIH may not reliably experience or report other symptoms of increased ICP. ${ }^{4}$ In addition, Digre and Corbett found that men require surgical intervention for IIH more frequently than women? While in our study men only required a change in management in 3 of 9 encounters, shunt revision was performed in all 3 cases. Ultimately, our findings suggest that males may present to the emergency department with more serious complaints that require admission and/or physicians have a lower threshold for admission with males, due to their greater risk of severe vision loss.

Performance of a diagnostic LP was also an important independent predictor of admission in our study, with patients at 3.10 greater odds for admission than for visits without an LP performed. In our study, no patient experienced complications related to diagnostic or therapeutic LPs. Thus, performance of an LP may be one of the most useful procedures in determining which patients should be admitted from the emergency department. Interest- 
ingly, the opening pressure was significantly lower in the admitted group (median $24 \mathrm{~cm} \mathrm{H}_{2} \mathrm{O}$ ) than the discharged group (median $30 \mathrm{~cm} \mathrm{H}_{2} \mathrm{O}$ ). However, this may be due to the fact that patients in the discharged group typically had their elevated ICP managed via shunt reprogramming, change in medication, or therapeutic CSF drainage, while the admitted patients were significantly more likely to require inpatient shunt revision surgery. Importantly, the fact that an LP was performed in the emergency department may indicate that there was a higher level of concern that the patient had a shunt-related problem, which may skew the interpretation of the analysis in our retrospective study. As such, the vast majority $(53,88 \%)$ of patients who received an LP also had additional LPs performed during at least one subsequent visit. Also of note, only 7 (12\%) of the patients who received an LP also had papilledema on funduscopic examination, highlighting the individual utility of an LP in the decision-making process for admission. Additionally, shunt patency examinations were also more frequently performed in admitted patients; however, this result is likely biased by our institution's common practice to admit patients who receive a shunt patency examination overnight.

Interestingly, patients with more prior emergency department visits were less likely to be admitted from the emergency department or undergo shunt revision, even after correcting for potential confounders in a multivariable analysis. Only 7 of $68(10 \%)$ visits in patients with a history of 10 or more prior emergency department visits resulted in admission compared with 31 of $103(30 \%)$ visits in patients with fewer than 10 prior emergency department visits. This may suggest that physicians have a higher threshold for admission for patients who more frequently present for symptoms related to IIH and these patients may require shunt revision less often.

A subanalysis found several factors associated with shunt revision in our study. For example, the presence of papilledema was an independent risk factor for shunt revision, but was only marginally associated with admission. The observance of papilledema on ophthalmological examination occurred in only 14 of $169(8 \%)$ visits in our study, and this low frequency likely decreased our ability to detect an independent impact. Moreover, formal ophthalmological funduscopy was not performed in 77 (46\%) visits, likely due to less concern of shunt pathology or frequent repeat visits for the same complaint. Importantly, subjective visual complaints were not associated with admission, highlighting the importance of a formal ophthalmological examination in the assessment of shunt-treated patients who present to the emergency department for IIHrelated symptoms.

Few studies have investigated the impact of racial differences on IIH, with several series reporting African American race as a poor prognostic indictor. ${ }^{5}$ Interestingly, while race did not have an impact on the frequency of emergency department admissions in our study, Caucasians were significantly more likely to undergo shunt revision. The explanation for this finding is unclear and likely multifactorial. Ultimately, further studies are needed to evaluate the possible impact of race on shunt-related outcomes in patients with IIH.
Likewise, several series have found an association between systemic hypertension and poorer long-term outcomes in IIH patients. ${ }^{5,7}$ In this study, hypertension was significantly associated with shunt revision, but not emergency department admission. While the reason is unclear, uncontrolled hypertension is a significant risk factor for vision loss, and a lower threshold to treatment may exist in patients with a known hypertensive history. In addition, several commonly used blood pressure medications can affect ICP, and may influence the severity of symptoms in patients with IIH. Importantly, we only assessed for a history of systemic hypertension and did not evaluate whether patients were controlled via antihypertensive medications. Therefore, the influence of systemic hypertension on patients with IIH remains unclear.

The presence of a programmable shunt was suggestive as a protective factor against shunt revision on multivariable analysis. While other factors may influence the impact of a programmable shunt with regard to the need for admission or shunt revision in patients with IIH, the ability to change the shunt drainage setting without surgical intervention may be useful in their long-term management.

The limitations of this study should be carefully considered. These limitations include retrospective data collection, limited patient sample, single-institution experience, and a heterogeneous number of emergency department visits per patient. Additionally, it is possible that we were unable to capture all of our patients' emergency department visits because they may frequent the emergency department of multiple hospitals, leading to a bias in the results. Despite these limitations, we found several potential risk factors related to admission and management in shunt-treated adult patients with IIH. Well-designed, prospective studies are needed to further evaluate these risk factors among both shunt-treated and conservatively managed patients.

\section{Conclusions}

The decision to admit a patient from the emergency department for symptoms related to IIH is challenging, particularly for shunt-treated patients. In this study, factors such as male sex, younger age at presentation, fewer prior emergency department visits, and performance of a diagnostic LP were independent predictors of admission. In addition, papilledema was strongly predictive of the need for shunt revision. Knowledge of the factors associated with the need for admission and shunt revision is needed to aid clinicians and surgeons in the decision-making process.

\section{Acknowledgments}

The authors report the following financial support and industry affiliations. Dr. Elder: This article reflects the views of the author and should not be construed to represent the FDA's views or policies. Mrs. Carson: National Center for Research Resources and the National Center for Advancing Translational Sciences (NCATS) of the National Institutes of Health (Grant No. 1UL1TR001079). Dr. Goodwin: United Negro College Fund-Merck Postdoctoral Fellow and an Award from the Burroughs Wellcome Fund. Dr. JusuéTorres: Salisbury Hydrocephalus Research Fellowship grant and the Swenson Family Foundation. Dr. Rigamonti: Salisbury Family Foundation and Swenson Family Foundation. 


\section{References}

1. Andrews LE, Liu GT, Ko MW: Idiopathic intracranial hypertension and obesity. Horm Res Paediatr 81:217-225, 2014

2. Ball AK, Clarke CE: Idiopathic intracranial hypertension. Lancet Neurol 5:433-442, 2006

3. Ball AK, Howman A, Wheatley K, Burdon MA, Matthews $\mathrm{T}$, Jacks AS, et al: A randomised controlled trial of treatment for idiopathic intracranial hypertension. J Neurol 258:874881,2011

4. Bruce BB, Kedar S, Van Stavern GP, Monaghan D, Acierno $\mathrm{MD}$, Braswell RA, et al: Idiopathic intracranial hypertension in men. Neurology 72:304-309, 2009

5. Bruce BB, Preechawat P, Newman NJ, Lynn MJ, Biousse V: Racial differences in idiopathic intracranial hypertension. Neurology 70:861-867, 2008

6. Dandy WE: Intracranial pressure without brain tumor: diagnosis and treatment. Ann Surg 106:492-513, 1937

7. Digre KB, Corbett JJ: Pseudotumor cerebri in men. Arch Neurol 45:866-872, 1988

8. Elder BD, Sankey EW, Goodwin CR, Jusué-Torres I, Khattab MH, Rigamonti D: Outcomes and experience with lumbopleural shunts in the management of idiopathic intracranial hypertension. World Neurosurg 84:314-319, 2015

9. Friedman DI, Jacobson DM: Diagnostic criteria for idiopathic intracranial hypertension. Neurology 59:1492-1495, 2002

10. Friesner D, Rosenman R, Lobb BM, Tanne E: Idiopathic intracranial hypertension in the USA: the role of obesity in establishing prevalence and healthcare costs. Obes Rev 12:e372-e380, 2011

11. Hamdallah IN, Shamseddeen HN, Getty JL, Smith W, Ali MR: Greater than expected prevalence of pseudotumor cerebri: a prospective study. Surg Obes Relat Dis 9:77-82, 2013

12. Jones JS, Nevai J, Freeman MP, McNinch DE: Emergency department presentation of idiopathic intracranial hypertension. Am J Emerg Med 17:517-521, 1999

13. Koerner JC, Friedman DI: Inpatient and emergency service utilization in patients with idiopathic intracranial hypertension. J Neuroophthalmol 34:229-232, 2014
14. Liu A, Elder BD, Sankey EW, Goodwin CR, Jusué-Torres I, Rigamonti D: Are shunt series and shunt patency studies useful in patients with shunted idiopathic intracranial hypertension in the emergency department? Clin Neurol Neurosurg 138:89-93, 2015

15. Liu A, Elder BD, Sankey EW, Goodwin CR, Jusué-Torres I, Rigamonti D: The utility of computed tomography in shunted patients with idiopathic intracranial hypertension presenting to the emergency department. World Neurosurg 84:18521856, 2015

16. Raoof N, Sharrack B, Pepper IM, Hickman SJ: The incidence and prevalence of idiopathic intracranial hypertension in Sheffield, UK. Eur J Neurol 18:1266-1268, 2011

\section{Disclosures}

The authors report no conflict of interest concerning the materials or methods used in this study or the findings specified in this paper.

\section{Author Contributions}

Conception and design: Elder, Goodwin, Rigamonti. Acquisition of data: Elder, Sankey, Liu, Jusué-Torres. Analysis and interpretation of data: Elder, Sankey, Liu. Drafting the article: Elder, Sankey, Liu, Goodwin. Critically revising the article: Elder, Carson, Goodwin, Jusué-Torres, Rigamonti. Reviewed submitted version of manuscript: all authors. Approved the final version of the manuscript on behalf of all authors: Elder. Statistical analysis: Elder, Sankey, Liu, Carson, Jusué-Torres. Study supervision: Elder, Goodwin, Rigamonti.

\section{Correspondence}

Benjamin Elder, Department of Neurosurgery, Johns Hopkins University School of Medicine, 1800 Orleans St., Rm. 6007, Baltimore, MD 21287. email: belder4@jhmi.edu. 